\title{
Hotspot Components for Gesture-Based Interaction
}

\author{
Alejandro Jaimes and Jianyi Liu \\ FXPAL Japan, Fuji Xerox Co., Ltd. \\ alex.ajimes@fujixerox.co.jp
}

\begin{abstract}
We present a novel camera-based adaptable user interface system that uses hotspot components for 2D gesture-based interaction. A camera points to the desktop and the image captured by the camera appears on the user's screen. A hotspot area is activated when a user's hand passes through the rectangle that defines it. For example, a right_left hotspot activates when the user moves his hand from right to left, entering and exiting the rectangle. Our system is highly flexible because it allows the user to customize the interface as follows: (1) hotspot areas can be created anywhere within the camera-captured image; (2) new commands can be assigned to particular hotspots or composite hotspot sequences (e.g., right_left for previous webpage; up+right+down for webpage reload); (3) a physical workspace on the desktop can be defined by pointing the camera to any location; (4) different hotspot layouts for different applications can be created and saved. The system works with an inexpensive webcam in real time and uses machine learning to automatically detect skin areas for robust gesture recognition.
\end{abstract}

\section{Introduction}

Lower hardware costs and higher computational power (webcams are standard in many new PCs and laptops) make camera-based interaction techniques promising. Gesture-based interaction is of particular interest because humans use gestures to communicate naturally, but defining a set of meaningful and computationally recognizable 3D gestures can be difficult. Two-dimensional gestures, on the other hand, can be easily defined, assigned application or user-dependent meanings, and recognized using simple computer vision techniques.

In this paper, we present a novel camera-based, adaptable user interface system that uses hotspot components for 2D gesture-based interaction. In our approach, a camera points to the user's desktop. The user defines an interaction area within the desktop by pointing the camera to a desired location, defines new commands by configuring hotspot areas, and executes them by moving his hand across them. For example, to move to the previous page in a document, the user moves his hand from left to right across a hotspot area (Fig. 1). The framework has many applications, as the desk can become a large interactive space in which a rich set of hotspot configurations can be created with different meanings. For instance, imagine an instrument in which each hotspot or hotspot configuration represents a different sound; a desk with multiple displays so that different parts of the desk can be used to simultaneously interact with multiple documents; hand gestures similar to "mouse gestures" for browsing; or special gestures for people with disabilities. 
Work related to our system includes frameworks where physical devices are used as interface components [3], tangible interfaces [5], and systems that learn objects for interaction [2]. We do not use physical devices as in [35] and the system does not learn new interface objects for programmers as in [2]. The framework of [9] uses modular "Visual Interface Cues" and Hidden Markov Models to learn gesture dynamics. Our approach does not require training (except for the skin filter), and focuses on allowing the user to combine simple hotspot components for desktop gesture recognition. Most gesture recognition approaches [67] image the entire hand to model different postures. Instead, we use small hotspot areas that respond only to simple 2D actions (e.g., entered from right to left, etc.). The framework in [1] is also based on the idea of camera-based interaction, but three types of widgets are used: button, linear, and circular. We use only one camera, and interaction is based on 2D configurations of simple, directional hotspot components. A similar framework was presented in [4], but using skin and face detection for video indexing.

The advantages of our system can be summarized as follows: (1) individual components can be very robust and easily combined for different purposes; (2) users do not need to learn complex gestures (simple 2D motion gestures suffice); and (3) users do not need to remember gestures (hotspots are visible on the screen).

\section{The User Interface}

In our system, a camera is placed above the desktop (for example, on top of the computer monitor) and the corresponding video captured by the camera appears in a resizable window on the computer screen (Fig. 1). The user defines the interface area by
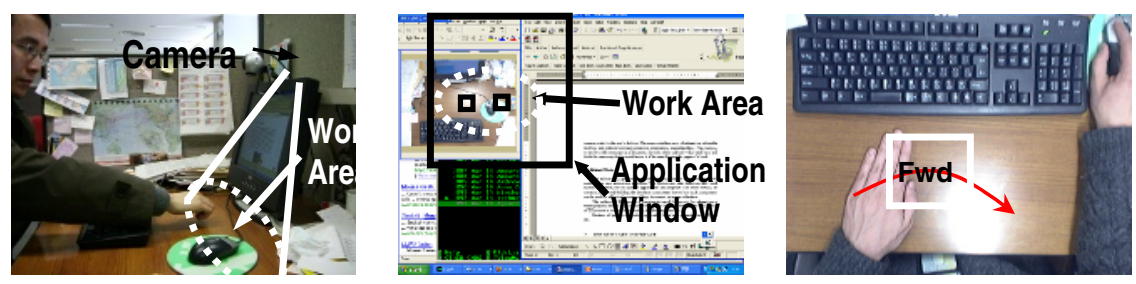

Fig. 1. System setup. The camera (left) points at a physical work area on the desktop. The interface image as viewed by the user (middle) can be resized as desired and shows the captured video images and hotspots (right image).
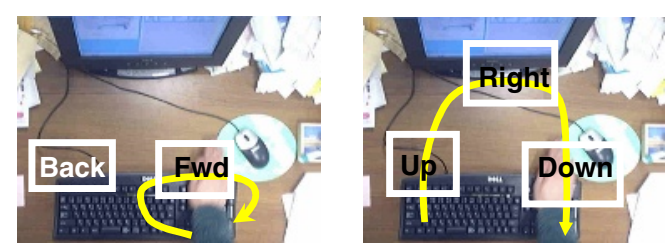

Fig. 2. Screen shot of captured image. On the right image a composition of hotspots represents a single command (e.g., re-load a webpage), whereas on the left each hotspot represents a different command (back, forward). 
moving the camera to point at a desired physical location: in Fig. 1 (left) the main capture area is behind the keyboard-a user might prefer to point the camera elsewhere (e.g., in front of the keyboard as in Fig. 1, right).

A hotspot area is a $2 \mathrm{D}$ area in the video image that is activated when the user moves his hands or fingers across the area in a particular direction. In the application window (Fig. 1, middle) the user can draw new hotspot rectangles and assign commands either to individual hotspots or combinations. Fig. 2 (right) shows a composite sequence of hotspots combined to represent a single action (e.g., reload a webpage). Such configurations can be saved, so the user could have a library of configurations for different applications if desired.

\section{Gesture Recognition}

Our recognition approach is based on the idea of simple and easily re-configurable 2D hotspot components: a gesture is recognized as a sequence of hotspot area activations. This approach yields effective and robust results because the gestures that each individual hotspot recognizes are very specific. This contrasts with approaches that image the entire hand or hands and use algorithms to disambiguate between different hand postures and movements.

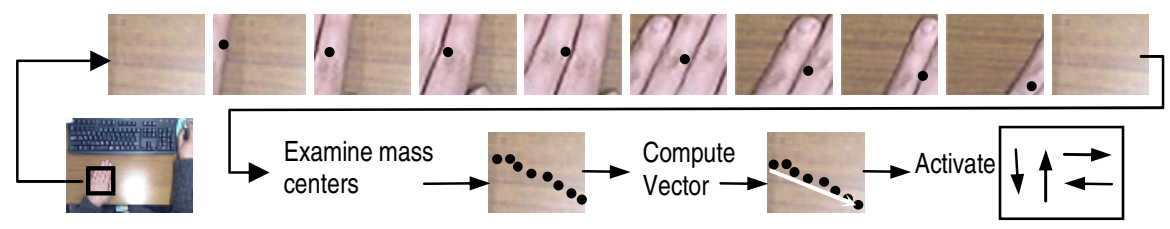

Fig. 3. Hotspot activation starts when a skin area is detected (frame 2) and ends when no skin is present (frame 10). In each sample, skin is detected and the center of mass is computed.

Hotspots are rectangular areas on a video image, defined by four coordinate points $(x 1, y 1, x 2, y 2)$, and an activation constraint. A hotspot is activated only when its activation constraint is met. Our system includes a basic library of $2 \mathrm{D}$ directional activation constraints: a hotspot is activated when a skin area enters the hotspot and exits it in one of four directions: left_to_right, right_to_left, top_to_bottom, and bottom_to_top. In order to increase robustness, a hotspot is assigned only one constraint. For example, the hotspot of 0 Fig. 1 (right) activates only if a skin area enters it from the left and exits on the right. We use a skin filter so that hotspots are activated only when hands/limbs are used to perform the gestures. The skin filter can be trained for each user (to increase robustness), or a standard one trained by the system developers can be used. The hotspot activation process, described next, is depicted in Fig. 3.

- Skin filter construction: first, we obtain a set of skin images and a set of images that do not contain skin. We convert each image to HSV color space (because it is perceptually uniform: points that are close in HSV space are also perceptually similar). This yields a training set of skin and non-skin pixels. We use a machine learning algorithm from Weka [8] to build a skin classifier. 
- Skin detection: the area corresponding to each hotspot is processed every $t$ milliseconds. In order to make processing more efficient, we examine only a subset of the pixels inside each hotspot rectangle: a pixel every $d$ pixels is classified into skin or not-skin using the skin filter. Then we compute the center of mass of the detected skin pixels within the hotspot area (black spots in Fig. 3).

- Hotspot activation: activation of a hotspot starts with the first frame in which skin pixels are found (hand enters hotspot) and ends when no skin pixels are found (hand leaves hotspot). This yields a sequence of center of mass points. We use the first $n$ points in the sequence to compute a motion start point, and the last $m$ points to compute a motion end point. Using the two points we estimate the motion vector. The hotspot is activated only if the vector's direction corresponds to the hotspot's constraint.

- Gesture recognition: a gesture is detected when all of its corresponding hotspots are activated in a particular sequence (e.g., Fig. 2, right).

We have performed quantitative skin detection experiments. Using 12 training images we obtain approx. 32,000 pixels. This yields the following results (using separate training (66\%) and testing sets (34\%)): Naïve Bayes [8] yields $90.23 \%$ accuracy, and 1 -Nearest Neighbor [8] yields $98.6 \%$ accuracy (with $d=n=m=2$ ). Quantitative evaluation of the activations or gestures is difficult since the results depend on the speed of the hand motions and on whether the user's hand properly enters the hotspot areas. However, we have found that individual hotspots are accurate close to $100 \%$ of the time (as long as the speed of the hand motion is reasonable).

\section{Conclusions and Future Work}

We presented a novel camera-based adaptable user interface system that uses hotspot components for 2D gesture-based interaction. The proposed component-based framework to interaction has two advantages: (1) combinations of simple components can yield higher accuracy than what may be achieved with gesture recognition frameworks that require segmentation and tracking, and (2) it allows high user customization so the user can create several hotspot configurations and save them for a wide range of different applications. Since the user views the image being captured by the camera, remembering specific gestures for particular applications is not necessary.

Future work includes integrating the prototype we have built with other applications, investigating ways to determine hotspot area sizes, integration with audio commands (e.g., to select different hotspot templates), experiments with users, and implementation on mobile devices (e.g., camera phones).

\section{References}

1. J. Alan, D.R. Olsen, "Light Widgets: Interacting in Every-day Spaces," in proc. ACM 7th Intl. Conference on Intelligent User Interfaces 2002, San Francisco, CA, Jan. 2002.

2. J.A. Fails, D.R. Olsen, "A Design Tool for Camera-based Interaction," CHI: ACM Conf. on Human Factors in Computing Systems, CHI Letters 5(1), pp. 449 - 56, 2003. 
3. S. Greenberg and C. Fitchett, "Phidgets: easy development of physical interfaces through physical widgets," in proc. UIST 2001, pp. 209-218, Orlando, Florida, Nov. 11-14, 2001.

4. Jaimes, Q. Wang, N. Kato, H. Ikeda, and J. Miyazaki, "Visual Trigger Templates for Knowledge-Based Indexing," in ACM/IEEE PCM 2004, Toyko, Japan, Dec. 2004.

5. S.R. Klemmer J.Li, J. Lin, and J.A. Landay, "Papier-Mâché: Toolkit Support for Tangible Input," in proc. ACM CHI 2004, Vienna, Austria, April 24-29, 2004.

6. S. Marcel, "Gestures for multi-modal interfaces: A Review," T.R. IDIAP-RR 02-34, 2002.

7. V.I. Pavlovic, R. Sharma and T.S. Huang, "Visual interpretation of hand gestures for human-computer interaction: a review", IEEE Trans. on PAMI, 19(7):677-695, 1997.

8. I.H. Witten and E. Frank, Data Mining: Practical Machine Learning Tools and Techniques with Java Implementations, Morgan Kaufmann, 1999.

9. G. Ye, J.J. Corso, D. Burschka, and G.D. Hager, "Vics: A modular hci framework using spatio-temporal dynamics," Machine Vision and Applications, 16(1):13-20, 2004. 